\title{
Co-contraction in RA patients with a mobile bearing total knee prosthesis during a step-up task
}

\author{
Eric H. Garling · Nienke Wolterbeek · Sanne Velzeboer · Rob G. H. H. Nelissen • \\ Edward R. Valstar · Caroline A. M. Doorenbosch · Jaap Harlaar
}

Received: 30 August 2007/ Accepted: 31 March 2008/Published online: 14 May 2008

(C) The Author(s) 2008

\begin{abstract}
It was hypothesized that rheumatoid arthritis (RA) patients with a total knee prosthesis that allows axial rotation of the bearing (MB) will show more co-contraction to stabilize the knee joint during a step-up task than RA patients with a fixed bearing total knee prosthesis $(\mathrm{FB})$ where this rotational freedom is absent while having the same articular geometry. Surface EMG, kinematics and kinetics about the knee were recorded during a step-up task of a MB group $(n=5)$, a FB group $(n=4)$ and a control group $(n=8)$. Surface EMG levels of thigh muscles were calibrated to either knee flexion or extension moments by means of isokinetic contractions on a dynamometer. During the step-up task co-contraction indices were determined from an EMG-force model. Controls showed a higher active ROM during the step-up task than the patient group, $96^{\circ}$ versus $88^{\circ}$ $(P=0.007)$. In the control group higher average muscle extension, flexion and net moments during single limb
\end{abstract}

E. H. Garling · N. Wolterbeek $(\bowtie)$.

R. G. H. H. Nelissen · E. R. Valstar

Department of Orthopaedics, Leiden University Medical Center,

PO Box 9600, J11-S, 2300 RC Leiden, The Netherlands

e-mail: n.wolterbeek@lumc.nl

E. H. Garling

Department of Radiology, Division of Image Processing,

Leiden University Medical Center, Leiden, The Netherlands

E. R. Valstar

Department of Biomechanics,

Faculty of Mechanical Engineering,

Delft University of Technology,

Delft, The Netherlands

S. Velzeboer · C. A. M. Doorenbosch · J. Harlaar

Department of Rehabilitation Medicine,

VU University Medical Center,

Amsterdam, The Netherlands support phase were observed than in the patient group. During the $20-60 \%$ interval of the single limb support, MB patients showed a significant higher level of flexor activity, resulting in a lower net joint moment, however co-contraction levels were not different. Compared to the control group arthroplasty patients showed a $40 \%$ higher level of co-contraction during this interval $(P=0.009)$. Control subjects used higher extension moments, resulting in a higher net joint moment. Visual analysis revealed a timing difference between the MB and FB group. The FB group seems to cocontract approximately 20\% later compared to the MB group. RA patients after total knee arthroplasty show a lower net knee joint moment and a higher co-contraction than controls indicating avoidance of net joint load and an active stabilization of the knee joint. MB and FB patients showed no difference in co-contraction levels, although timing in FB is closer to controls than MB subjects. Since visual analysis revealed a timing difference between the $\mathrm{MB}$ and $\mathrm{FB}$ group, this may express compensation by coordination. Rehabilitation programs for RA patients should include besides muscle strength training, elements of muscle-coordination training.

Keywords Mobile bearing knee - Knee stability .

Co-contraction - Total knee arthroplasty .

Rheumatoid arthritis

\section{Introduction}

The aim of total knee replacement is relief of pain and functional improvement. The two most commonly implanted total knee designs are the fixed bearing (FB) posterior stabilized (PS) total knee and the mobile bearing (MB) total knee prosthesis. 
The fixed bearing PS total knee prosthesis was designed to provide passive stability for the knee joint [1, 25, 30]. The post and cam interaction stabilizes the joint in mediallateral direction and facilitate femoral rollback when the knee is flexed.

Mobile bearing total knee prostheses have polyethylene inserts that can rotate and/or translate with respect to the tibial plateau. Therefore, a MB total knee has less internal stability and depends more upon preserved ligaments and active structures to provide stability of the knee joint compared to a FB total knee design. It has been shown that joint instability can lead to high levels of muscle co-contraction of agonist and antagonist muscle groups surrounding the knee [2].

Many clinical, biomechanical and modeling studies support the hypothesis about higher levels of co-contraction of the quadriceps and hamstrings during dynamic tasks to provide an active stabilization of the knee to compensate for the loss of passive structures e.g. the cruciate ligaments after total knee arthroplasty [6, 8, 9, 21, 23, 26-29]. The use of surface EMG is an independent technique to assess co-contraction, but is hindered by the complex relation between muscle force and EMG. However, EMG-to-force processing can be applied in dynamic tasks, such as a step-up, when combining an EMG-to-activation model with a (physiologic) muscle model of muscle kinematics [20]. It has also been shown that sub maximal contractions can be used to calibrate EMG to force [16], which makes this technique applicable to patients after total knee arthroplasty [18].

In this study it was hypothesized that subjects with a total knee prosthesis that allows axial rotation of the bearing will show more co-contraction to stabilize the knee joint during a step-up task than subjects with a FB total knee prosthesis where this rotational freedom is absent while having the same articular geometry.

\section{Methods}

\section{Subjects}

The power calculation for the number of subjects in this study is based on the study of Doorenbosch and Harlaar [14]. In that study, five controls were compared with five anterior cruciate ligament deficient subjects and they found a significant difference in co-contraction index (CCI) between the two groups. The mean CCI for patients was 0.54 (SD 0.04) versus a CCI of 0.25 (SD 0.07) for the controls. Based on this information a sample size of nine patients versus eight controls would be sufficient to detect a difference of 0.05 between controls and patients. Unfortunately, no literature is available about differences in CCI between two prosthesis groups.
Therefore in this study, nine patients suffering from rheumatoid arthritis (RA) were included in our specialized rheumatoid arthritis clinic approximately 6 months after total knee arthroplasty. The institutional medical-ethical committee approved the study and all subjects gave informed consent. In five patients, a MB NexGen Legacy Posterior Stabilised (MB group) prosthesis was implanted and in four patients a FB NexGen Legacy Posterior Stabilised (FB group), (Zimmer Inc, Warsaw, USA). As a control group, eight healthy persons were selected who had no functional impairment of any lower extremity joint. For the control group, the data of the non-dominant leg was acquired. The "non-dominant" leg for the controls was chosen for comparability, assuming that patients with a total knee prosthesis preferred the non-operated leg.

The tibial articular surfaces of the MB group are made of net-shape moulded UHMW polyethylene. The tibial bearing component is snapped onto an anterior-centrally located trunnion at the polished cobalt chromium base plate, which prevents tilting and determines the center of rotation of the bearing. The slot in the plastic allows for $25^{\circ}$ of internal-external rotation of the mobile bearing, limited by an anterior bar. In the FB group, this rotational freedom of the tibial bearing is absent.

For both prosthesis groups, the cam of the femoral component engages the tibial spine at approximately $75^{\circ}$ and induces mechanical rollback while inhibiting posterior subluxation of the tibia. In the frontal plane, the component has a dished articulation, providing a large contact area even up to $7^{\circ}$ varus/valgus mal alignment. In addition to the cam/spine mechanism, the femoral component has a large distal radius and smaller posterior radius to help facilitate femoral rollback on the tibia during lower flexion angles.

Inclusion criteria for the prosthesis groups for the study were the ability to perform a step-up without the help of bars or a cane, the ability to walk more than $1 \mathrm{~km}$, not use walking aids, symptom less with no apparent functional impairment of any other lower extremity joint besides the operated knee and no or slight pain during activity according to the Knee Society Pain Score [17]. Furthermore, they had to have a unilateral total knee replacement. Prior to the experiment anthropometric data was assessed for all three groups (Table 1).

\section{Experimental protocol}

The subjects performed the step-up task barefoot, in a smooth and linear manner at a self-selected, comfortable speed. At the beginning of the step-up, the patient was asked to stand, feet together, at a distance of $5 \mathrm{~cm}$ in front of the 18-cm-high platform, and step onto the platform using the limb with the implant under investigation first. 
Table 1 Subjects data and kinetic parameters for the MB knee group $(n=5)$, FB group $(n=4)$, the combined patient group $(n=9)$ and control group $(n=8)$ during the single limb support phase and 20-60\% interval of the single limb support phase

\begin{tabular}{|c|c|c|c|c|c|c|}
\hline & $\begin{array}{l}\text { MB group median, } \\
\text { min-max }\end{array}$ & $P$ & $\begin{array}{l}\text { FB group median, } \\
\text { min-max }\end{array}$ & $\begin{array}{l}\text { Patient group median, } \\
\text { min-max }\end{array}$ & $P$ & $\begin{array}{l}\text { Control group median, } \\
\text { min-max }\end{array}$ \\
\hline Age (years) & $64,46-74$ & ns & $67,60-81$ & $66,46-81$ & 0.002 & $30,19-54$ \\
\hline BMI $\left(\mathrm{kg} / \mathrm{m}^{2}\right)$ & $30,21-34$ & ns & $28,22-32$ & $29,21-34$ & ns & $23,20-32$ \\
\hline $\operatorname{Sex}(F / M)$ & $4 / 1$ & ns & $1 / 2$ & $5 / 3$ & ns & $4 / 4$ \\
\hline Side (L/R) & $2 / 3$ & ns & $3 / 0$ & $5 / 3$ & ns & $1 / 7$ \\
\hline Duration (s) & $2,1.8-2.4$ & ns & $2,2.1-2.4$ & $2,1.8-2.4$ & ns & $2,1.9-2.5$ \\
\hline $\operatorname{ROM}\left(^{\circ}\right)$ & $87,64-92$ & ns & $90,84-95$ & $88,64-95$ & 0.007 & $96,89-106$ \\
\hline \multicolumn{7}{|l|}{ Single limb } \\
\hline $\mathrm{CCI}$ & $0.6,0.4-0.7$ & ns & $0.6,0.5-0.7$ & $0.6,0.4-0.7$ & ns & $0.5,0.3-0.7$ \\
\hline Mext (Nm) & $17,12-20$ & ns & $18,17-20$ & $17,12-20$ & 0.003 & $25,17-61$ \\
\hline Mflex (Nm) & $-28,-30$ to -27 & ns & $-18,-43$ to 16 & $-28,-43$ to -16 & 0.012 & $-17,-25$ to -6 \\
\hline Mnet (Nm) & $-12,-15$ to -8 & ns & $0,-26$ to 4 & $-12,-26$ to 4 & 0.005 & $9,-1$ to 54 \\
\hline \multicolumn{7}{|c|}{$20-60 \%$ single limb } \\
\hline CCI & $0.7,0.6-0.8$ & ns & $0.7,0.7-0.8$ & $0.7,0.6-0.8$ & 0.009 & $0.5,0.2-0.8$ \\
\hline Mext (Nm) & $24,22-31$ & ns & $28,28-30$ & $28,22-31$ & 0.001 & $44,32-105$ \\
\hline Mflex (Nm) & $-32,-43$ to -27 & 0.025 & $-21,-24$ to -14 & $-28,-43$ to -14 & ns & $-15,-36$ to -6 \\
\hline Mnet (Nm) & $-10,-18.2$ to 4 & 0.049 & $7,4-17$ & $-1.4,-18.2$ to 17 & 0.005 & $27,3-98$ \\
\hline
\end{tabular}

The step-up was finished when the contralateral foot was placed on top of the riser. After a brief orientation session, the patient performed at least three step-ups with a maximum of five, with a rest period of 2 min between trials. In all cases an assistant was near the patient during the measurements for safety reasons. During the step-up task knee kinematics, EMG of thigh muscles and ground reaction forces were measured.

\section{Calibration of the EMG force processing}

Prior to the step-up task, the EMG levels were calibrated towards mechanical units using an isokinetic dynamometer (Kin-Com 500 H, Chattex Corp, Chattanooga, TE, USA). All subjects were instructed to exert maximal isokinetic knee flexion and extension contractions. However it was not necessary to actually perform maximum force as submaximal forces are sufficient for normalization [16]. During the experiments, subjects were seated with their hips flexed at $90^{\circ}$ of flexion. The trunk and upper leg of the subject were rigidly fixated to the chair. A part of the seat was especially designed with a hole, to keep the electrodes at the dorsal side of the thigh free and prevent contact artefacts. The projection of the knee axis of flexion and extension at the lateral condyle was aligned with the rotation axis of the dynamometer. The rotatable arm of the dynamometer was fixed to the tibia at a distal position. The dynamometer angle offset was set to reflect on an anatomical knee angle, defined by the line of lateral malleolus, knee axis and greater trochanter.

For the calibration, concentric isokinetic flexion and extension contractions were performed at three different velocities $\left(30,60,90, \mathrm{deg} \mathrm{s}^{-1}\right)$. Contractions were randomly ordered and rest pauses of 2 min were between each of them. The exerted moment, processed EMG signals, range of motion and angular velocity were recorded $(100 \mathrm{~Hz})$ during each isokinetic flexion and extension movement of the knee.

\section{Electromyography}

Surface EMG electrodes (Meditrace $\mathrm{Ag}-\mathrm{AgCl}$; lead-off area $1 \mathrm{~cm}^{2}$; center-to-center distance $2.5 \mathrm{~cm}$ ) were used to record the activation of five thigh muscles. EMG of the following muscles were recorded: $\mathrm{m}$. rectus femoris; $\mathrm{m}$. vastus lateralis; $\mathrm{m}$. vastus medialis; $\mathrm{m}$. semitendinosus; $\mathrm{m}$. biceps femoris $\mathrm{c}$. longum. The electrodes were placed longitudinally over the muscle bellies after standard preparation of the skin [15]. A reference-electrode was placed on a bony part of the shank.

Surface EMG was recorded by a bipolar lead-off and online removal of artefacts by high pass filtering at $20 \mathrm{~Hz}$. Simultaneously, the EMG signals were shown on screen for on line visual inspection to check for undesirable coactivation during the calibration contractions. Offline, the EMG signals were rectified and low pass filtered at $2 \mathrm{~Hz}$ to obtain the EMG envelopes.

\section{Kinematics and kinetics}

During the step-up task, the vertical and horizontal components of the ground reaction forces and moments during the step-up were recorded by means of a force plate 
(AMTI, Boston, MA, USA) and sampled at 1,000 Hz. From these signals, the magnitude, direction and point of application of the force vector were calculated.

Simultaneously, the 3D kinematics was assessed with an optoelectronic motion analysis system (Optotrak: Northern Digital Inc, Canada) at a frame rate of 100 frames per second. A three segment-model was used including the upper leg, lower leg and foot. To define local coordinate systems of the lower leg and the upper leg, a triangle at each segment containing three light-emitting diodes (LED's) was attached with straps. The third triangle defining the foot segment was attached with tape on the instep of the foot. With a stylus anatomical landmarks were defined relatively to the local coordinate system of the triangle into an anatomical coordinate system: trochanter major, lateral femur condyle, medial femur condyle, tuberositas, caput fibulae, lateral malleolus, medial malleolus, lateral side of the foot on the fifth metatarsal, medial side of the foot on the first metatarsal and the calcaneus.

Kinematics in the sagittal plane were also obtained with a video camera operating at 25 frames per second for visual inspection of undesirable postural compensation strategies.

\section{Data analysis}

The start of the movement cycle (0\%) was defined as the first change $\left(>5^{\circ}\right)$ in knee angle of the leg of interest. The end of the movement cycle $(100 \%)$ was when the contralateral foot was also placed on top of the riser and the change in knee angle of the leg of interest was zero. The co-contraction index (CCI) was determined during the single limb support phase. Although commonly in gait analysis the single limb support phase starts when the other leg is off the ground, the single limb support phase in this study starts on the first moment of weight loading on the platform. This phase ends at the last moment of single limb support on the top of the platform determined by the onset of the ground reaction force moving medially on the platform.

An EMG-force model was used to calculate muscle moments and the CCI. This model has been thoroughly validated [14-16]. In general, the isokinetic measurements are used to include length and velocity influences on the EMG to force relation, to obtain estimated moments of agonists and antagonist muscles (Magonist, Mantagonist) separately.

To quantify the amount of co-contraction or active stabilization, Magonist and Mantagonist were used in defining the CCI according to $[14,16]$

$\mathrm{CCI}=1-\left\{\frac{[(\text { Magonist })-(\text { Mantagonist })]}{[(\text { Magonist })+(\text { Mantagonist })]}\right\}$

The CCI ranges between 0 and 1 . CCI values close to 1 indicate a high level of co-contraction of agonists and antagonists and a CCI value of 0 indicates a pure reciprocal activation. For each individual subject, the CCI was calculated as the mean value of the muscle moments during the single limb support phase of the step-up task.

Statistical analysis

A non-parametric Mann-Whitney $U$ Test was performed. Significance was accepted at an alpha level of $P<0.05$. All statistical computations are performed with a commercial statistical package (SPPS, SPSS, USA).

\section{Results}

The most important variables and p-values are listed in Table 1. Mean time after operation was 9.6 months (SD 3.5 months, range 5-17 months). The questionnaire showed that $38 \%$ of the patients declare their operated leg as their dominant leg. The duration of the step-up task was comparable for all groups. In addition, the phases defined during the step-up: foot-lift, foot-placement, double-stance and single limb support were similar between groups. Controls showed a higher active ROM during the step-up task then the patient group $(P=0.007)$.

In the control group higher average muscle extension, resulting in higher net moments, and higher flexion moments during single limb support phase was observed than in the patient group (Fig. 1). Since the control subjects used higher extension moments, this resulted in a higher net joint moment. No differences between the $\mathrm{MB}$ and FB group were observed. The differences between the FB group and controls for the variables muscle flexion moments, extension moments and net knee joint moments were smaller than between the MB group and controls.

In the interval from 20 to $60 \%$ of the single limb support, all individual subjects showed the peak muscle extension moment. In this interval there was a significant difference between the MB and FB group in the knee flexion moment and the net knee moments (respectively $P=0.025$ and $P=0.049$ ). The MB patients showed a significant higher level of flexor activity, resulting in a lower net joint moment. However, co-contraction levels were not different. A significant difference was found for co-contraction between the patient group and the control group (average CCI was respectively 0.7 and 0.5 , $P=0.009$ ). Visual analysis revealed a timing difference between the MB and FB group. The FB group seems to co-contract approximately 20\% later (first and second peak of the CCI) in the single limb support phase compared to the MB group (Fig. 2). 
Fig. $1 \mathrm{M}_{\text {flexion }}$ (dark grey), $\mathrm{M}_{\text {extension }}$ (light grey) and $\mathrm{M}_{\text {net }}$ (line) for all four groups (a control; b patient; c MB; d FB) during the entire single limb support phase
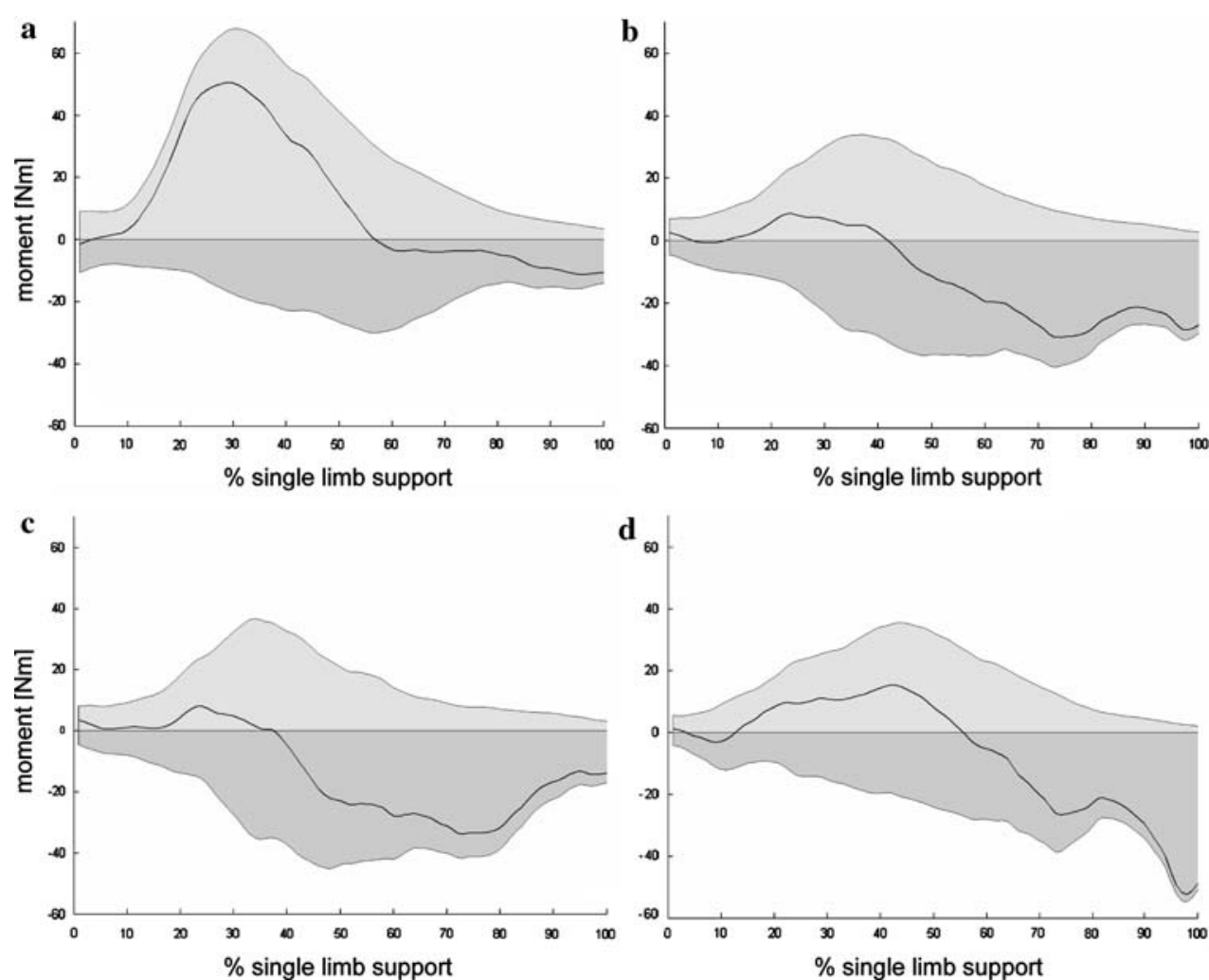

\section{Discussion}

In this study an EMG-force model has been used to address if there are differences in co-contraction between RA patients with a MB or FB total knee prostheses. Although timing in FB patients is closer to controls than MB subjects, the latter could not be confirmed during the step-up task. This might be caused by the small patient groups. However, there was a significant difference in co-contraction between the patient group and the control group. To increase the power of studies using an EMG-to-activation model in patients after total knee arthroplasty, larger patient groups are recommended. Also, a MB design which allows AP translation in addition to rotation might show more distinctive differences between the two designs.

In a previous study, maximal voluntary contraction was used to calibrate the EMG signals [18]. Avoidance for pain leads to an improper maximal activation of isolated muscles during isolated contractions. Only during daily activity tasks subjects are willing to give high activation levels. The new method used in the current study using an EMG-force model calibrated with sub-maximal contractions showed to be suitable for patients after TKA $[15,16]$. Although this method has proven to have a high discriminating power [14], differences between the two prostheses could not be observed during the step-up task.

In the study of Garling et al. [18] it was shown that subjects with a MB design show higher EMG levels

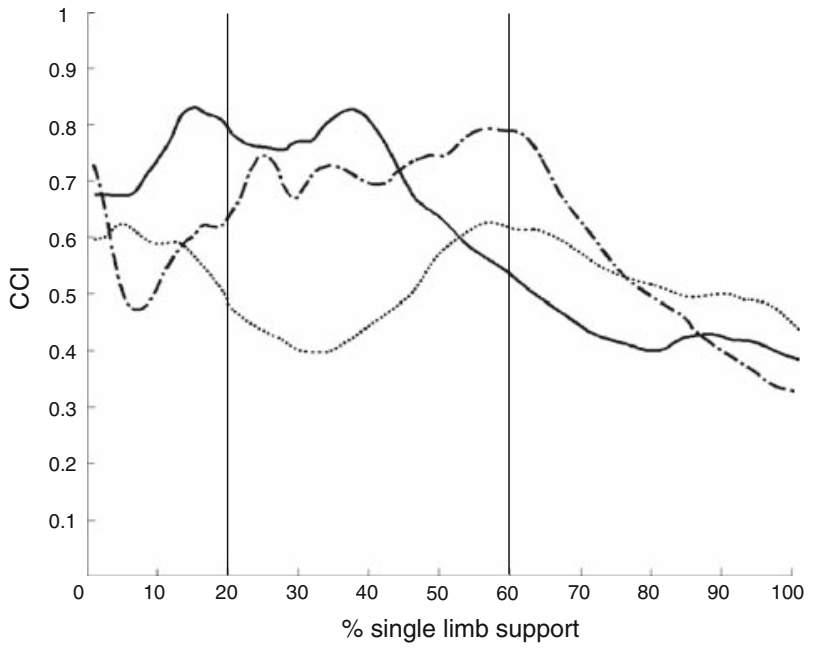

Fig. 2 CCI values for the MB group (line), the FB group (dashdotted) and the control group (dotted) during the single limb support phase. The $20-60 \%$ interval is also indicated

compared to subjects with a PS fixed bearing design. However, no difference in co-contraction was observed between the two groups. One of the differences between that study and the current study is the use of a MB design with more degrees of freedom of the inlay. The MB knee design in the previous study permits both anterior/posterior sliding as rotation of the inlay on the tibial tray. It can be expected that a MB that allows also anterior/posterior 
sliding of the inlay result in more co-contraction than the MB used in the current study that only allows axial rotation of the inlay. Tibiofemoral translations affect the quadriceps moment arm by changing the instantaneous center of rotation. Femoral rollback with flexion will increase the moment arm of the quadriceps. When an intrinsic AP constraint is absent, the hamstrings can be recruited as secondary AP stabilisers. Consequently, co-contraction will be increased.

Another explanation for the same amount of co-contraction between the two designs found in this study is the actual mobility of the mobile bearing inlay. It has been shown that the amount of axial rotation of the MB design used in the current study is very limited or even absent [19]. The kinematics of the inlay and consequently the tibiofemoral kinematics can be compared to a fixed bearing total knee design with the same articular geometry where no motion of the bearing occurs.

The FB group showed a peak co-contraction approximately $20 \%$ later during the stance phase than the MB group. In preparation for foot contact with the ground, an early hamstring activity stabilizes the knee [24]. The hamstrings pull the tibia into a position so that the knee joint is stable during extension. The patient group showed also a lower net knee joint moment and a higher co-contraction than controls indicating avoidance of net joint load and an active stabilization of the knee joint. In another study comparing a MB and a fixed bearing TKA design during stair ascending, a decrease in the frontal external knee moments in the MB group was observed suggesting a compensatory loading mechanism [11].

An abnormal negative net knee moment was found in the whole single limb support phase in the MB-group and FB-group. In the 20-60\% interval, only the MB-group has a negative net knee moment. The large muscle flexion moments are an explanation for this negative net knee moments. This would imply that flexion is accomplished while extension is actually performed. During analysis of the videotape made during step-up, it appeared that patients did not use another step-up strategy than the controls. However, even a slight forward lean (e.g. $3 \mathrm{~cm}$ ) of the patients' trunk would already explain this change in net joint moment. The same patterns for the net knee moment were found in other studies [3, 7, 11]. Another possibility for the large flexion moments is a neglect of the bi-articular nature of the hamstrings in our model. The force-length relationship of the muscles during measurements with the dynamometer assumes hip flexion. Hip extension during step up could influence the length dependence of the EMG to force model considerably.

Patellofemoral geometry has a significant effect on knee kinematics. Especially the quadriceps moments in the joint are dependent on the orientation of the prosthesis relative to the patella [3, 5]. Andriacchi and Hurwitz [3] evaluated two different groups of patients during stair climbing that only differed in the curvature of the femoral trochlea. The group with a design that had non-anatomical tracking of the patella had a higher than normal flexion moment of the knee during late stance phase. In the current study the patellofemoral kinematics are not explored but the results show resembling high flexion moments when extension is expected for the patients, without significant differences between the MB and the FB group.

Patients with rheumatoid arthritis have used medication for years, which has effect on bone strength and the function of soft-tissue surrounding the prosthesis. Although the other joints of the patients were symptom less and showed no functional impairment it cannot be guaranteed that the kinematics were not influenced.

Abnormal kinematics and eventual dysfunction of the prosthesis might be a result of the decreased bone and tissue quality [12]. Even in the most clinically successful cases of non-RA patients treated by total knee replacement cannot achieve normal joint function over time. In most cases gait remains slower than normal, muscle strength is decreased, less work is produced, the treated knee has limited ROM both during stance and the swing phase and muscle moments are changed [7, 10, 22]. Although other studies show comparable results with the current study regarding a decreased active ROM during step-up for the RA patients of about $10-15 \%$, without differences in duration of the step-up [4, 11, 13], co-contraction can be added to changes in joint function after total knee arthroplasty based on the findings of this study. Continuing follow-up of the patients after TKA should clarify whether the active stabilization of the knee joint is a lasting adaptation or changes over time.

Staircase data provides an approximation to other activities involving a flexed knee position under high load, such as sitting and rising from a chair or bed and using a toilet. Knee flexion and exerted moments are higher in activities like sitting and rising from a chair. Further research should therefore focus on other activities as well to describe possible functional differences between $\mathrm{MB}$ and FB total knee prostheses.

\section{Conclusion}

RA patients after total knee arthroplasty show lower net knee joint moments and higher co-contraction than controls indicating avoidance of net joint load and an active stabilization of the knee joint. The MB and FB groups show no differences in co-contraction levels, although coordination in patients with a FB is closer to controls than patients with a MB. Timing differences between the MB and FB group, may 
express compensation by coordination. Rehabilitation programs for RA patients should include besides muscle strength training, elements of muscle-coordination training.

Open Access This article is distributed under the terms of the Creative Commons Attribution Noncommercial License which permits any noncommercial use, distribution, and reproduction in any medium, provided the original author(s) and source are credited.

\section{References}

1. Aglietti P, Buzzi R, De Felice R, Giron F (1999) The InsallBurstein total knee replacement in osteoarthritis: a 10-year minimum follow-up. J Arthroplasty 14:560-565. doi:10.1016/S08835403(99) 90077-3

2. Alkjaer T, Simonsen EB, Jørgensen U, Dyhre-Poulsen P (2003) Evaluation of the walking pattern in two types of patients with anterior cruciate ligament deficiency: copers and noncopers. Eur J Appl Physiol 89:301-308. doi:10.1007/s00421-002-0787-x

3. Andriacchi TP, Hurwitz DE (1997) Gait biomechanics and the evolution of total joint replacement. Gait Posture 5:256-264

4. Andriacchi TP, Galante JO, Fermier RW (1982) The influence of total knee-replacement design on walking and stair-climbing. J Bone Joint Surg 64:1328-1335

5. Andriacchi TP, Yoder D, Conley A, Rosenberg A, Sum J, Galante JO (1997) Patellofemoral design influences function following total knee arthroplasty. J Arthroplasty 12(3):243-249. doi: 10.1016/S0883-5403(97) 90019-X

6. Baratta R, Solomonow M, Zhou BH, Letson D, Chuinard R (1988) D'Ambrosia R Muscular coactivation. The role of the antagonist musculature in maintaining knee stability. Am J Sports Med 16(2):113-122

7. Benedetti MG, Catani F, Bilotta TW, Marcacci M, Mariana E, Giannini S (2003) Muscle activation pattern and gait biomechanics after total knee replacement. Clin Biomech 18:871-876. doi:10.1016/S0268-0033(03) 00146-3

8. Boerboom AL, Hof AL, Halbertsma JP, van Raaij JJ, Schenk W, Diercks RL, van Horn JR (2001) Atypical hamstrings electromyographic activity as a compensatory mechanism in anterior cruciate ligament deficiency. Knee Surg Sports Traumatol Arthrosc 9(4):211-216. doi:10.1007/s001670100196

9. Bulgheroni P, Bulgheroni MV, Andrini L, Guffanti P, Giughello A (1997) Gait patterns after anterior cruciate ligament reconstruction. Knee Surg Sports Traumatol Arthrosc 5(1):14-21. doi: 10.1007/s001670050018

10. Byrne JM, Gage WH, Prentice SD (2002) Bilateral lower limb strategies used during a step-up task in individuals who have undergone unilateral total knee arthroplasty. Clin Biomech 17(8):580-585. doi:10.1016/S0268-0033(02) 00061-X

11. Catani F, Benedetti MG, De Felice R, Buzzi R, Giannini S, Aglietti P (2003) Mobile and fixed bearing total knee prosthesis functional comparison during stair climbing. Clin Biomech 18(5):410-418. doi:10.1016/S0268-0033(03) 00044-5

12. Chmell MJ, Scott RD (1999) Total knee arthroplasty in patients with rheumatoid arthritis. Clin Orthop Rel Res 366:54-60

13. Costigan PA, Deluzio KJ, Wyss UP (2002) Knee and hip kinetics during normal stair climbing. Gait Posture 16:31-37. doi: 10.1016/S0966-6362(01) 00201-6
14. Doorenbosch CAM, Harlaar J (2003) A clinically applicable EMG-force model to quantify active stabilization of the knee after a lesion of the anterior cruciate ligament. Clin Biomech 18:142-149. doi:10.1016/S0268-0033(02) 00183-3

15. Doorenbosch CA, Harlaar J (2004) Accuracy of a practicable EMG to force model for knee muscles. Neurosci Lett 368(1):7881. doi:10.1016/j.neulet.2004.06.055

16. Doorenbosch CA, Joosten A, Harlaar J (2005) Calibration of EMG to force for knee muscles is applicable with submaximal voluntary contractions. J Electromyogr Kinesiol 15(4):429-435. doi:10.1016/j.jelekin.2004.11.004

17. Ewald FC (1989) The Knee Society total knee arthroplasty roentgenographic evaluation and scoring system. Clin Orthop Relat Res 9:

18. Garling EH, van Eck M, Wedding T, Veeger DJ, Valstar ER, Nelissen RG (2005) Increased muscle activity to stabilise mobile bearing knees in patients with rheumatoid arthritis. Knee 12(3):177-182. doi:10.1016/j.knee.2004.07.005

19. Garling EH, Kaptein BL, Nelissen RGHH, Valstar ER (2007) Limited rotation of the mobile bearing in a rotating platform total knee prosthesis. J Biomech 40:S25-S30

20. Hof AL, Pronk CAN, Best JA (1987) Comparison between EMG to force processing and kinetic analysis for the calf muscle moment in walking and stepping. J Biomech 20:167-187

21. Imran A, O'Connor JJ (1998) Control of knee stability after ACL injury or repair: interaction between hamstrings contraction and tibial translation. Clin Biomech 13(3):153-162. doi:10.1016/ S0268-0033(97) 00030-2

22. Kaufman KR, Hughes C, Morrey BF, Morrey M, An KN (2001) Gait characteristics of patients with knee osteoarthritis. J Biomech 34:907-915. doi:10.1016/S0021-9290(01) 00036-7

23. Kellis E (1998) Quantification of quadriceps and hamstring antagonist activity. Sports Med 25(1):37-62

24. Lass P, Kaalund S, leFevre S, Arendt-Nielsen L, Sinkjaer T, Simonsen O (1991) Muscle coordination following rupture of the anterior cruciate ligament. Electromyographic studies of 14 patients. Acta Orthop Scand 62(1):9-14

25. Li PL, Zamora J, Bentley G (1999) The results at ten years of the Insall-Burstein II total knee replacement. Clinical, radiological and survivorship studies. J Bone Joint Surg (Br) 81:647-653

26. O'Connor JJ (1993) Can muscle co-contraction protect knee ligaments after injury or repair? J Bone Joint Surg (Br) 75(1):4148

27. Pandy MG, Shelburne KB (1998) Theoretical analysis of ligament and extensor-mechanism function in the ACL-deficient knee. Clin Biomech 13(2):98-111. doi:10.1016/S0268-0033(97) 00055-7

28. Roberts CS, Rash GS, Honaker JT, Wachowiak MP, Shaw JC (1999) A deficient anterior cruciate ligament does not lead to quadriceps avoidance gait. Gait Posture 10(3):189-199. doi: 10.1016/S0966-6362(99) 00038-7

29. Shelburne KB, Pandy MG (1998) Determinants of cruciate-ligament loading during rehabilitation exercise. Clin Biomech 13(6):403-413. doi:10.1016/S0268-0033(98) 00094-1

30. Stern SH, Insall JN (1992) Posterior stabilized prosthesis: Results after follow-up of nine to twelve years. J Bone Joint Surg (Am) 74A:980-986 